\title{
FARMÁCIA VIVA: CULTIVO E MANEJO DE PLANTAS MEDICINAIS NO HORTO FLORESTAL, CUIABÁ-MT, BRASIL
}

Nhaára Da Vila Pereira ${ }^{1}$

Margô De David ${ }^{2}$

Maria Corette Pasa ${ }^{3}$

\section{INTRODUÇÃO}

O uso das espécies vegetais com fins de tratamento e cura de doenças é conhecido desde as antigas civilizações (CARVALHO; SARTI, 2004). Entre os itens que compõem o meio natural, as plantas se constituem como elemento principal para a fabricação de fitoterápicos e outros medicamentos convencionais e são utilizadas como remédios caseiros em práticas populares. Fato este se deve aos estudos etnofarmacológicos, que em sua prática agrega os valores de uso empírico das espécies vegetais com o respaldo científico que buscam legitimar os efeitos farmacológicos existentes nos mesmos (GONÇALVES, 2015).

Para a Organização Mundial de Saúde (OMS), as plantas consideradas como medicinais precisam conter, em um ou mais de seus órgãos, substâncias que possam ser usadas com propósitos terapêuticos ou que sejam precursoras de síntese de fármacos (WHO, 1979).

O Brasil tem ampla tradição no uso de plantas medicinais e tem tecnologia para validar cientificamente este conhecimento e ressalta que, cerca de $80 \%$ da população dos países em desenvolvimento utiliza derivados de plantas medicinais para suas necessidades de atenção primária de saúde (BRASIL, 2011). Uma vez que, o expressivo uso dos recursos naturais, para fins terapêuticos se deve, principalmente, à extensa e diversificada flora (DE DAVID; PASA, 2015).

1 Bióloga pela UFMT. Membro DGP ESCER/CNPq/UFMT. MT. nhaara.bio2012@gmail.com

2 Profa. Doutoranda pelo PPG Bionorte. MT. margodedavid@hotmail.com

3 Dra. PPG em Ciências Florestais e Ambientais. UFMT. Cuiabá. MT. pasaufmt@gmail.com 
O SUS oferece serviços que envolvem a produção e uso de plantas medicinais, de drogas vegetais e seus derivados e/ou de fitoterápicos, normatizados em legislação específica em esferas municipais e estaduais, sendo alguns implementados há mais de dez anos (GIRÃO; RODRIGUES, 2005). O Projeto Farmácia Viva é um exemplo desta extensão. Liderado pelo professor Francisco José de Abreu Matos da Universidade Federal do Ceará, o projeto, organizado sob influência da OMS, tem características de um programa de medicina social. Segundo Matos (1998, p. 17):

“...sua finalidade é oferecer, sem fins lucrativos, assistência farmacêutica fitoterápica às comunidades onde haja carência de atendimento dos programas de saúde pública, promovendo o uso correto de plantas de ocorrência local ou regional, dotadas de atividade terapêutica cientificamente comprovada".

Considerando-se as plantas medicinais importantes instrumentos da Assistência Farmacêutica, no final da década de 70, a Organização Mundial da Saúde (OMS) criou o Programa de Medicina Tradicional, objetivando o desenvolvimento de estudos científicos para melhor elucidar a qualidade e eficácia desses. Contudo, somente em 2006, foi aprovada, a Política Nacional de Plantas Medicinais e Fitoterápicos (PNPMF) e a Política Nacional de Práticas Integrativas (PNPIC), no Sistema Único de Saúde (SUS). Estas políticas trazem como diretrizes, dentre outras ações, a implantação/adequação de ações e serviços de medicina tradicional chinesa/acupuntura, homeopatia, plantas medicinais e fitoterapia (BRASIL, 2011).

A demanda pela utilização por parte das plantas medicinais está relacionada diversos fatores, dentre os quais, o alto custo dos medicamentos sintéticos e a falta de acesso da população à assistência médica e farmacêutica, e a tendência dos consumidores em utilizar produtos de origem natural (SIMÕES et al 1988). Desta forma, ao fazer o levantamento etnofarmacobotânico das espécies pertencentes à etnocategoria de uso medicinal presente no viveiro Horto Florestal em Cuiabá - MT, desde o manejo à sua produção in natura, ressaltando suas finalidades, formas de usos e as indicações terapêuticas é visível o propósito científico da Farmácia Viva para a sociedade em geral. 


\section{DESENHO EXPERIMENTAL}

A pesquisa foi conduzida na unidade de conservação do Horto Florestal - Tote Garcia, criado através da Lei no 5.053 de 28 de dezembro de 2007 de acordo com a Política Nacional de Práticas Integrativas e Complementares do SUS. Se localiza no bairro Balneário São Joaquim, em Cuiabá-MT. O Horto está situado na zona Sul da cidade, e encontra-se delimitado pelas coordenadas geográficas " $15^{\circ} 37$ ' 35 19" S e 56 03' 24 34" O. (Marsaro et al., 2013), conforme Figura 1.

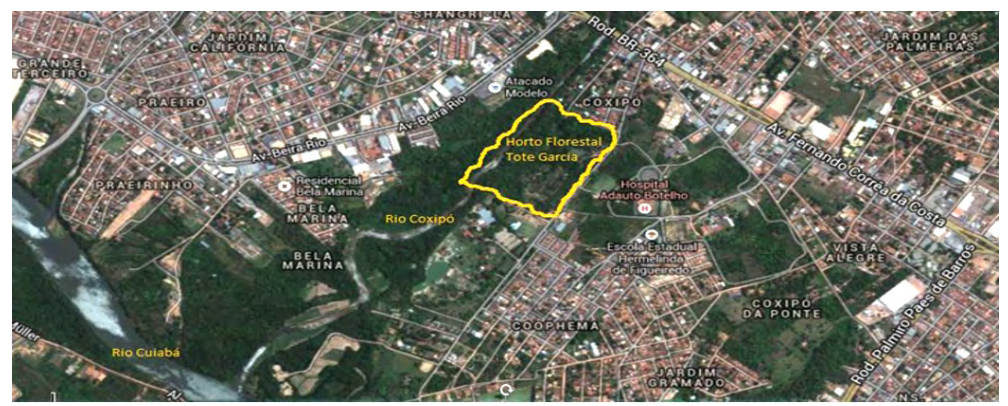

Figura 1. Área de estudo do Horto Florestal - Parque Tote Garcia, Cuiabá- MT. 2017. Fonte: Google Maps.

Os dados foram coletados entre o período de Maio e Junho de 2017, com frequência semanal no local de estudo. Os sujeitos participantes da pesquisa foram os profissionais do Núcleo de Fitoterapia e Plantas Medicinais - Fitoviva e a comunidade visitante do Horto. O procedimento metodológico aborda tratamentos Qualitativo e Quantitativo, envolvendo as seguintes técnicas: Entrevistas dos tipos semiestruturada e fechada (Minayo,1994), diário de campo, história oral (Meihy, 2010) e turnê guiada. Durante a coleta de dados será utilizada máquina fotográfica digital para armazenar imagens que envolvam o manejo com as plantas na área de estudo. Para a análise quantitativa será utilizado o Nível de Fidelidade (NF), Fator de Correção (FC) e o Pcup (\%). Esses cálculos expressam o consenso informante e permitem avaliar a importância relativa de cada planta, segundo Friedman (1986); Amorozo e Gelly (1988); Rossato et al. (1999); Luoga (2000); Albuquerque e Andrade (2002); Pasa, (2007; 2008; 2010; 2011; 2012), através da equação: 


$$
\boldsymbol{N} \boldsymbol{F}=\frac{F i d}{F s p} X 100
$$

Nível de Fidelidade (NF) consiste na razão entre o número de informantes que sugeriram o uso de uma espécie para uma finalidade maior (Fid) pelo número total de informantes que mencionaram a planta para algum uso (Fsp), multiplicado por 100.

$$
\boldsymbol{F C}=\frac{F s p}{I C E M C}
$$

O Fator de Correção (FC) é dado pela razão entre o número total de informantes que mencionaram determinada planta para algum uso (Fsp) pelo número da finalidade de uso mais citada.

$$
\boldsymbol{P c u p}=N F \times F C
$$

Pcup (\%) que tem por finalidade apresentar a frequência relativa do uso de cada espécie citada é operacionalizado pelo produto do Nível de Fidelidade (NF) com o Fator de Correção (FC).

\section{RESULTADOS E DISCUSSÃO}

\section{Perfil dos depoentes}

Foram entrevistadas 16 pessoas, sendo a maioria do sexo feminino (75\%) sendo quatro do sexo masculino. Dados semelhantes foram encontrados pelos seguintes autores (PASA, 2007; DE DAVID; PASA, 2015; DE DAVID, 2015, MOREIRA; GUARIM-NETO, 2015), fato que justifica serem delegados às mulheres os cuidados com a saúde da família e preparo dos remédios caseiros.

A idade entre os informantes variou de 32 a 60 anos. Dentre os entrevistados $44 \%$ possui o ensino médio, $31 \%$ ensino fundamental, $25 \%$ superior e/ou possuem formação em alguma área técnica.

Referindo-se ao estado de saúde destes informantes e seus hábitos citaram algumas doenças crônicas, como diabetes e hipertensão arterial. Do total de entrevistados, $65 \%$ não praticam 
nenhum tipo de atividade física. Quanto à assistência à saúde 75 $\%$ dos depoentes possui algum tipo de plano de saúde.

A religião católica obteve $35 \%$, seguida da espírita com $30 \%$, na sequência a evangélica com $20 \%$ e $15 \%$ relataram não possuírem religião. Gonçalves (2016), em seu trabalho na comunidade de Sucuri, Cuiabá, MT também destacou que a maioria dos informantes era católica. (Figura 2).

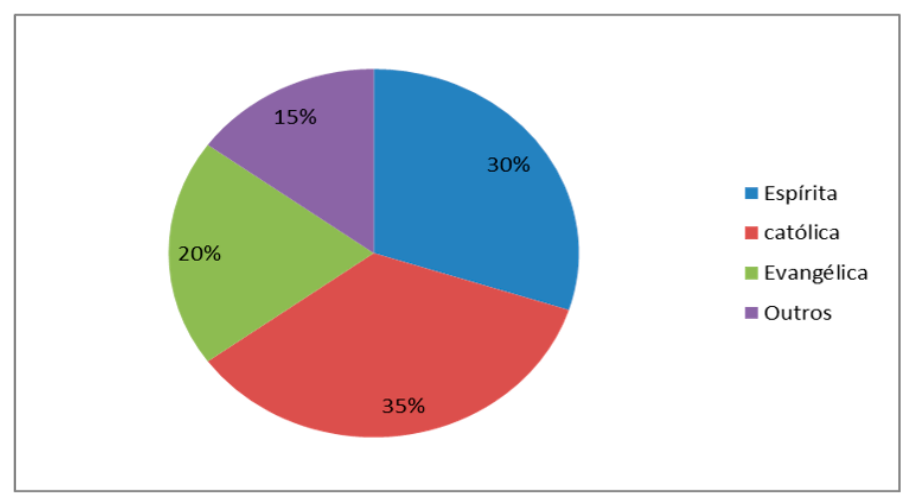

Figura 2 - Religião dos entrevistados. Cuiabá-MT, 2017

Fonte: Construção dos autores

\section{Dados etnofarmacobotânico}

A Tabela 1 apresenta as 48 espécies cultivadas no viveiro do Horto Florestal distribuídas em 30 famílias botânicas. Pode-se observar que as famílias botânicas de maior representatividade foram: Lamiaceae (nove espécies), Asteraceae (oito espécies), Zingiberaceae (quatro espécies). Casagrande (2009) encontrou resultados semelhantes na sua pesquisa pela comunidade Morro da Cruz, Porto Alegre - RS. Segundo De David (2015) também encontrou as famílias, Asteraceae e Lamiaceae, como as mais expressivas em sua pesquisa com os quintais urbanos de Várzea Grande, Mato Grosso.

\begin{tabular}{lr}
\hline Nome Científico/Família & Nome Vernacular \\
\hline Acanthaceae & \\
Justicia pectoralis Leonard & Anador / chambá \\
\hline
\end{tabular}




\section{Anacardiaceae}

Anacardium occidentale L.

Caju

Myracrodruon urundeuva Allemão

Aroeira

\section{Amaranthaceae \\ Chenopodium ambrosioidia Standl \\ Erva de santa maria \\ Pfaffia glomerata (Spreng.) Pedersen \\ Ginseng brasileiro}

\section{Alismataceae}

Echinodorus grandiflorus

Chapéu-de-couro

(Cham. E Schltdl.) Micheli

\section{Arecaceae}

Oenocarpus bacaba Mart.

Babaçu

\begin{tabular}{lc}
\hline Asteraceae & \\
Artermisia absinthum L. & Losna \\
Bacharis trimera Less. & Carqueja \\
Mikania glomerata Spreng. & Guaco \\
Solidago microglossa Meyen & Arnica-de-jardim/ Arnica-brasileira \\
Vernonia polyanthes (Spreng.) Less. & Caferana \\
\hline
\end{tabular}

\section{Bignoniaceae}

Arrabidea chica Lohmann

Crajirú

\section{Bixaceae}

Bixa orellana L.

Urucum

\section{Celastraceae}

Maytenus aquifolium Mart.

Espinheira santa

\section{Costaceae}

Costus spicatus (Jacq.) Sw. 


\section{Cucurbitaceae}

Momordica charantia L.

Melão de são caetano

Crassulaceae

Bryophyllum pinnatum (Lam.) Oken

Fortuna

\section{Euphorbiaceae}

Croton salutares Muelle. Arg. Baill.

Sangra d'água

\section{Caesalpiniaceae}

Copaifera langsdorffii St.-Hil.

Copaíba

\section{Fabaceae}

Amburana cearensis (Allemão) A.C. Sm

Amburana

Lauraceae

Persea americana Mill.

Abacate

\section{Lamiaceae}

Mentha pulegium (Mill.)

Poejo

Mentha arvensis L.

Vique

Mentha $x$ villosa Huds.

Hortelã comum

Ocimum basilicum L.

Alfavaca

Plectranthus barbatus Andrews

Boldo comum

Rosmarinus officinalis $\mathrm{L}$.

Alecrim

Hyptis suaveolens (L) Poit.

Erva-cidreira

Plectranthus amboinicus Sprengel, Curt

Hortelã-boldo

Plectranthus ornatus Codd

Boldinho, Boldo-pequeno

\section{Malpighiaceae}

Malpighia emarginata DC.

Acerola

Continua... 


\section{Myrtaceae}

Psidium guajava $\mathrm{L}$.

Goiaba

Eugenia uniflora L.

Pitanga

\section{Moraceae}

Morus nigra L.

Amora

\section{Passifloraceae}

Passiflora edulis Sims

Maracujá

Plantaginaceae

Plantago major L.

Tansagem

\section{Phyllanthaceae}

Phyllanthus niruri L.

Quebra-pedra

\section{Poaceae}

Cymbopogon citratus (DC.) Stapf

Capim cidreira/ capim-limão

\section{Polygonaceae}

Polygonum punctatum Elliot

Erva de Bicho

\section{Salicaceae}

Casearia sylvestris Sw.

Guaçatonga

\section{Solanaceae}

Solanum paniculatum $\mathrm{L}$.

Jurubeba

\section{Rutaceae}

Ruta graveolens $\mathrm{L}$.

Arruda

\section{Aloaceae}

Aloe vera (L.) Burm. $\mathrm{f}$.

Babosa

Continua... 
Zingiberaceae

Alpinia zerumbet (Pers.) B.L.

Burtt \& R.M. Sm.

Colônia

Zingiber officinale Roscoe

Gengibre

Curcuma longa $\mathrm{L}$.

Açafrão da terra

Curcuma zedoaria Roscoe William

Zedoária

Tabela 1. Espécies de uso Medicinal no Viveiro do Horto Florestal. Cuiabá- MT, 2017

Em relação às plantas utilizadas como remédio pelos entrevistados, foi identificado um total de 25 espécies distribuídas em 16 famílias. As famílias botânicas que apresentaram o maior número de citações de uso foram: Lamiaceae e Acanthaceae, sendo que diversas plantas foram indicadas para sintomas e sinais relativos ao sistema digestório, respiratório, excretor, circulatório, reprodutor feminino, nervoso, e ainda como vermicidas, anti-inflamatório e para dores no corpo, conforme apresentadas na Tabela 2.

As espécies foram sequenciadas de acordo com a sua importância relativa de usos principais (Pcup), apresentando a seguinte ordem: Justicia pectoralis Jacq. - Anador; Mentha arvensis L. - Hortelã comum (Pcup 56\% cada); Bacharis trimera L. - Carqueja (Pcup 33\%); Ocimum gratissimum L. - Alfavaca e Plectranthus barbatus A. - Boldo comum (Pcup 22\%). Corroborando com os resultados, De David (2015) em sua pesquisa com os quintais urbanos de Várzea Grande, MT, encontrou resultados semelhantes de frequência relativa de concordância para anador - Justicia pectoralis Jacq. e boldo - Plectranthus barbatus A. (60\% cada).

Dentre os vários epítetos, "chambá" e "anador" são os mais empregados para Justicia pectoralis Jacq. (Figura 3) pertencente à família Acanthaceae, nativa do Norte do Brasil (OLIVEIRA; ANDRADE, 2000). Esta espécie se encontra no Registro Nacional de Plantas Medicinais de Interesse para o SUS (Ministério da Saúde, 2008). Trata-se de uma pequena erva perene com até $40 \mathrm{~cm}$ de altura, folhas simples membranáceas e flores de coloração mariscada, estreitas e longas é uma planta bastante difundida pelo mundo. É usado popularmente contra reumatismo, cefaleia, febre, cólicas abdominais, inflamações pulmonares, tosse e também como expectorante, sudorífica e afrodisíaca. Dentre os vários com- 
ponentes registrados em sua análise fitoquímica, a cumarina e a umbeliferona são as principais. A avaliação realizada com extrato hidroalcoólico de folhas de "chambá" comprovaram suas atividades antipiréticas, analgésicas, espasmolíticas, anti-inflamatório e broncodilatadora (LORENZI; MATOS, 2000).

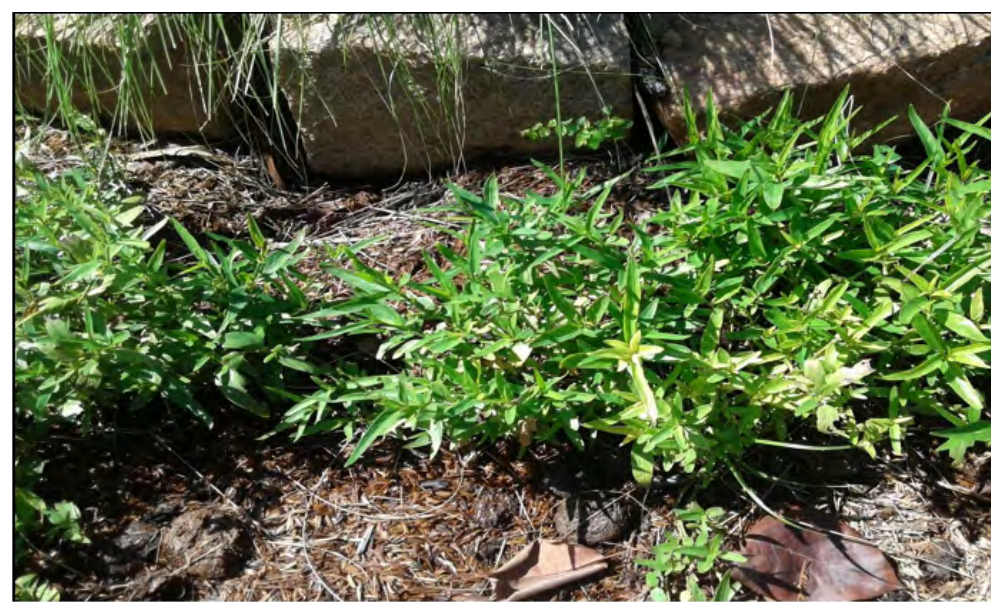

Figura 3. Justicia pectoralis. Horto Florestal, MT. 2017

Fonte: Construção dos Autores

A Farmacopéia do Brasil inclui sob a designação de hortelã, as folhas e ramos floridos de Mentha arvensis conhecida também por menta, hortelã japonesa ou brasileira (figura 4). Seu cultivo inicial se deu no Oriente, na china e Coreia, após a 1a guerra Mundial estabeleceu-se no Brasil (COSTA, 2002). Toda a parte aérea da planta é utilizada como medicinal. De acordo com a literatura etnofarmacológica, o uso medicinar popular atribui-lhe propriedades antidispéptica, antivomitiva, descongestionante nasal e antigripal. Estudo fitoquímicos registra como principal componente das folhas o óleo essencial contendo até $70 \%$ de $/$-mentol acompanhado de menores quantidades de outros álcoois, cetonas e hidrocarbonetos terpênicos. $O$ estudo farmacológico do óleo essencial destaca sua atividade contra bactérias e fungos colagogo (LORENZI; MATOS, 2000). De acordo com Souza et al (2004), a adição do óleo em concentrações específicas em meios de cultura com fungos fitopatógenos demonstrou a eficiência na inibição do desenvolvimento de organismos fitopatógenos. 


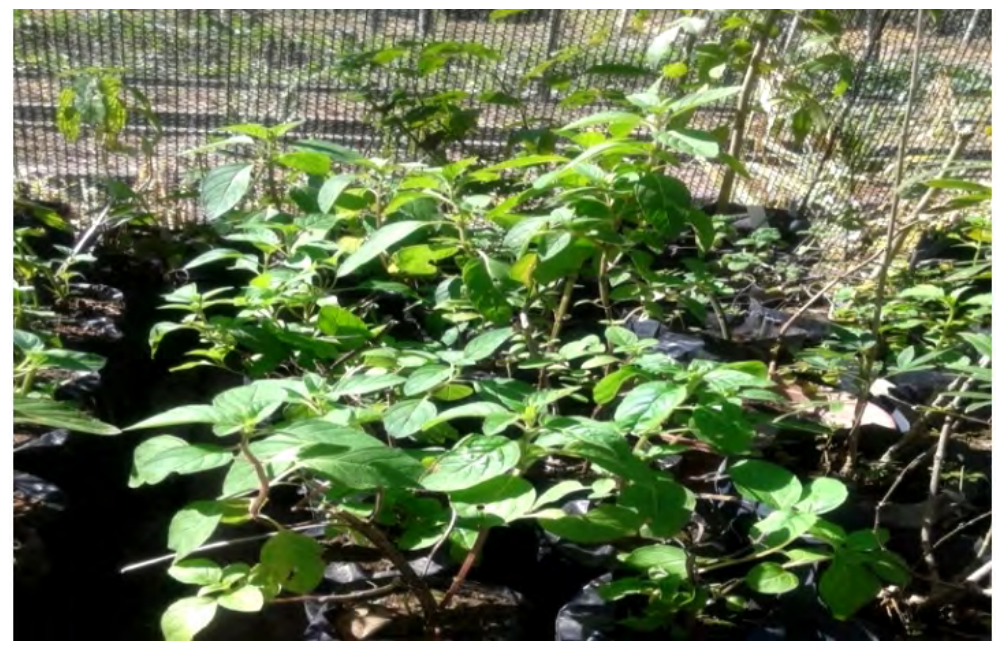

Figura 4. Mentha arvensis. Horto Florestal, MT. 2017

Fonte: Construção dos autores

Baccharis trimera pertencente à família Asteraceae, possui grande utilização na medicina tradicional e na produção de fitoterápicos (BORELLA ET AL., 2006). As diferentes propriedades atribuídas à carqueja na medicina tradicional vêm sendo estudadas e algumas foram validadas, como consequência dos resultados obtidos (LORENZI; MATOS 2002). As propriedades digestivas, anti-úlcera e antiácida foram validadas em estudos com cobaias, ao mostrar que os extratos da planta reduziram a secreção gástrica e tiveram efeito analgésico (GAMBERINI et al., 1991). Extratos alcoólicos de carqueja apresentam potencial antimicrobiano, conforme comprovaram Bara e Vanetti (1997), conforme Figura 5. 


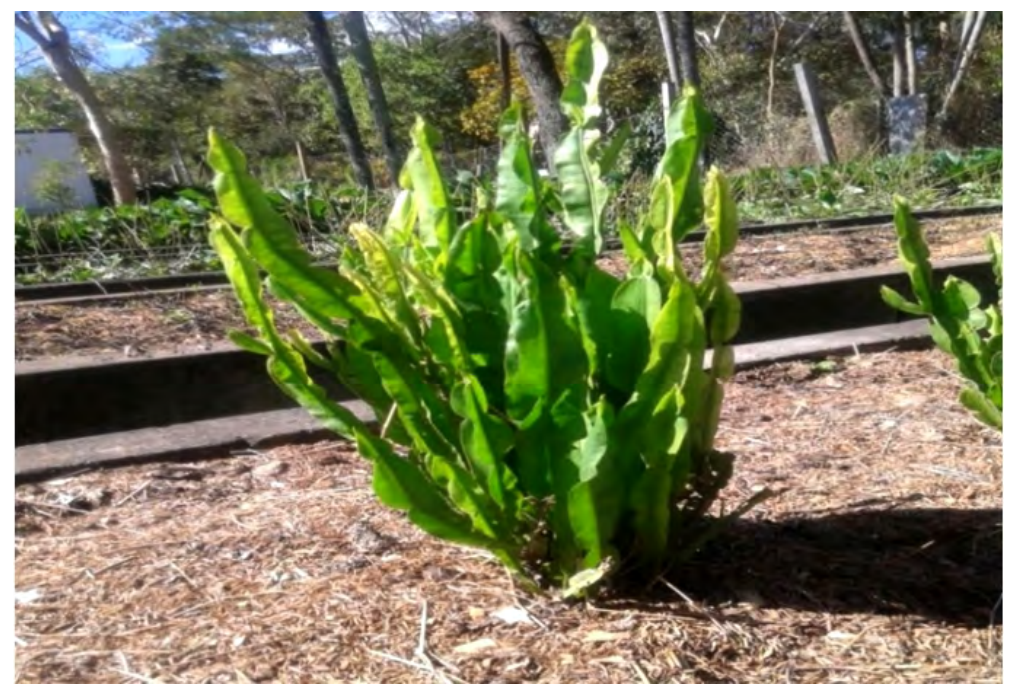

Figura 5. Baccharis trimera. Horto Florestal, MT. 2017

Fonte: Construção dos Autores.

A Lamiaceae é uma das famílias botânicas que apresenta propriedades medicinais devido à presença de flavonoides, alcaloides, taninos e os compostos fenólicos como o ácido rosmarínico e o ácido cafeíco que entre outras propriedades são antioxidantes (MARIUTTI; BRAGAGNOLO, 2007). De acordo com a literatura nas práticas usuais da medicina caseira as folhas de Ocimum gratissimum - alfavaca (Figura 6), são utilizadas na preparação de banhos antigripais, para tratar casos de nervosismo e paralisia, (LORENZI; MATOS, 2008). 


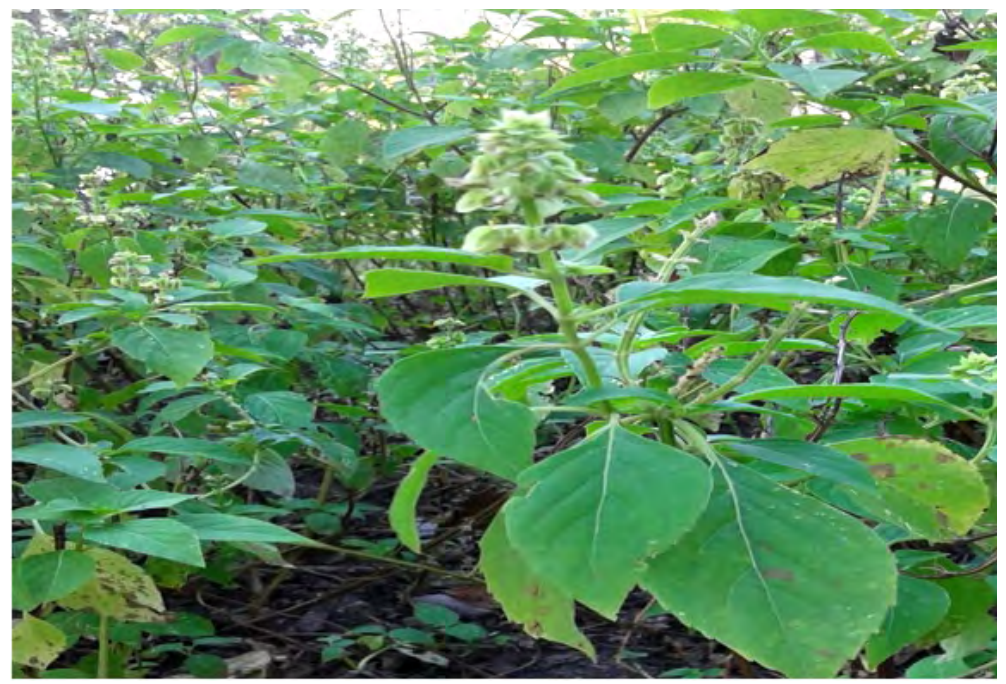

Figura 6. Ocimum gratissimum. Horto Florestal, MT. 2017

Fonte: Construção dos autores

A espécie Plectranthus barbatus (Figura 7), conhecida popularmente como boldo-comum ou boldo-do-brasil, é originária da Índia sendo amplamente cultivada em todo o Brasil, com utilidades na medicina populares e como fitoterápicas, por suas propriedades analgésicas e anti-dispéptica (CARRICONDE et al., 1996). A análise fitoquímica registra a presença de barbatusina, ciclobarbatusina, carocal, além de tripertenóides e esteroides e utilizada nos tratamentos de gastrite, dispepsia, azia, mal-estar gástrico (LORENZI; MATOS, 2002). 


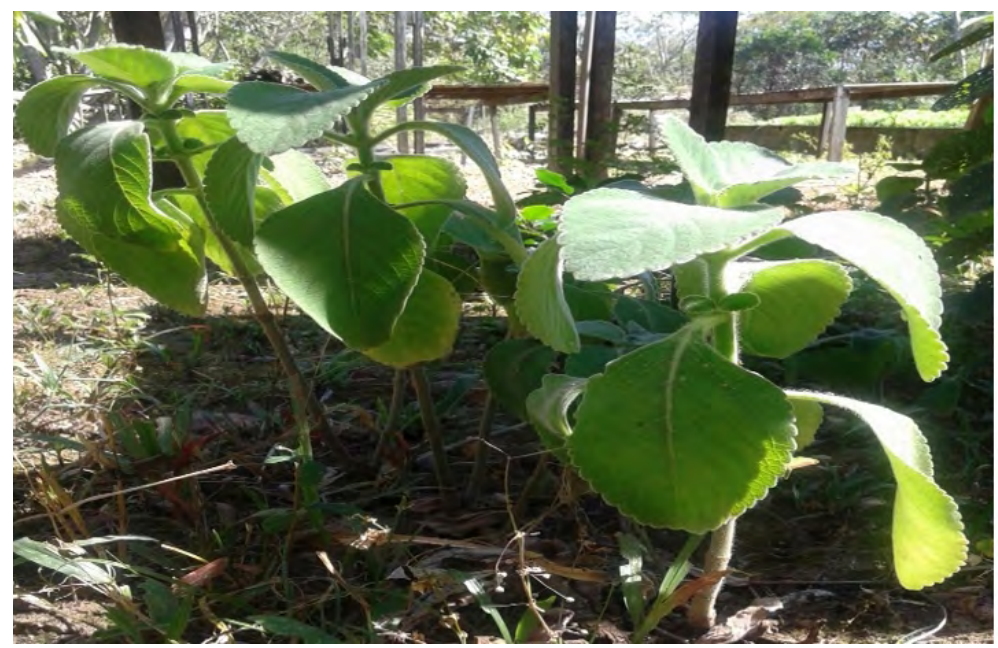

Figura 7. Plectranthus barbatus. Horto Florestal, MT. 2017

Fonte: Construção dos Autores.

\begin{tabular}{|c|c|c|c|c|c|c|c|c|c|}
\hline $\begin{array}{l}\text { Nome Científico/ } \\
\text { Familia }\end{array}$ & $\begin{array}{c}\text { Nome } \\
\text { vernacular }\end{array}$ & $\begin{array}{l}\text { Parte } \\
\text { Usada }\end{array}$ & $\begin{array}{l}\text { Formas } \\
\text { de Uso }\end{array}$ & $\begin{array}{l}\text { Uso mencio- } \\
\text { nado pelos } \\
\text { entrevistados }\end{array}$ & Fsp & Fid & NF & FC & Pcup(\%) \\
\hline \multicolumn{10}{|l|}{ Acanthaceae } \\
\hline $\begin{array}{l}\text { Justicia pectoralis } \\
\text { Leonard }\end{array}$ & $\begin{array}{l}\text { Anador / } \\
\text { chambá }\end{array}$ & Folha & $\begin{array}{c}\text { Chá e } \\
\text { infusão }\end{array}$ & $\begin{array}{c}\text { Dor de cabe- } \\
\text { ça, febre }\end{array}$ & 6 & 5 & 83 & 0,67 & $56 \%$ \\
\hline \multicolumn{10}{|l|}{ Amaranthaceae } \\
\hline $\begin{array}{l}\text { Chenopodium } \\
\text { ambrosioidia } \\
\text { Standl }\end{array}$ & $\begin{array}{l}\text { Erva de san- } \\
\text { ta maria }\end{array}$ & Folhas & $\begin{array}{l}\text { Chá e } \\
\text { curtido } \\
\text { no leite }\end{array}$ & $\begin{array}{c}\text { Febre, dor de } \\
\text { cabeça, dor } \\
\text { de estômago }\end{array}$ & 1 & 1 & 100 & 0,1 & $11 \%$ \\
\hline \multicolumn{10}{|l|}{ Asteraceae } \\
\hline $\begin{array}{l}\text { Bacharis trimera } \\
\text { Less. }\end{array}$ & Carqueja & $\begin{array}{l}\text { Folhas e } \\
\text { raízes }\end{array}$ & Chá & $\begin{array}{l}\text { Dor muscular, } \\
\text { inchaço, }\end{array}$ & 4 & 3 & 75 & 0,44 & $33 \%$ \\
\hline $\begin{array}{l}\text { Vernonia } \\
\text { polyanthes } \\
\text { (Spreng.) Less. }\end{array}$ & Caferana & Folhas & Chá & $\begin{array}{c}\text { Fígado/Icte- } \\
\text { rícia }\end{array}$ & 1 & 1 & 100 & 0,11 & $11 \%$ \\
\hline \multicolumn{10}{|l|}{ Bignoniaceae } \\
\hline $\begin{array}{l}\text { Arrabidea chica } \\
\text { Lohmann }\end{array}$ & Crajirú & Folhas & Chá & $\begin{array}{l}\text { Infecção no } \\
\text { útero, Corri- } \\
\text { mento } \\
\end{array}$ & 1 & 1 & 100 & 0,1 & $11 \%$ \\
\hline \multicolumn{10}{|l|}{ Celastraceae } \\
\hline $\begin{array}{l}\text { Maytenus } \\
\text { aquifolium Mart. }\end{array}$ & $\begin{array}{l}\text { Espinheira } \\
\text { santa }\end{array}$ & Folhas & $\begin{array}{l}\text { Chá, gar- } \\
\text { rafada }\end{array}$ & $\begin{array}{c}\text { Gastrite, } \\
\text { úlcera, quei- } \\
\text { mação }\end{array}$ & 1 & 1 & 100 & 0,1 & $11 \%$ \\
\hline
\end{tabular}




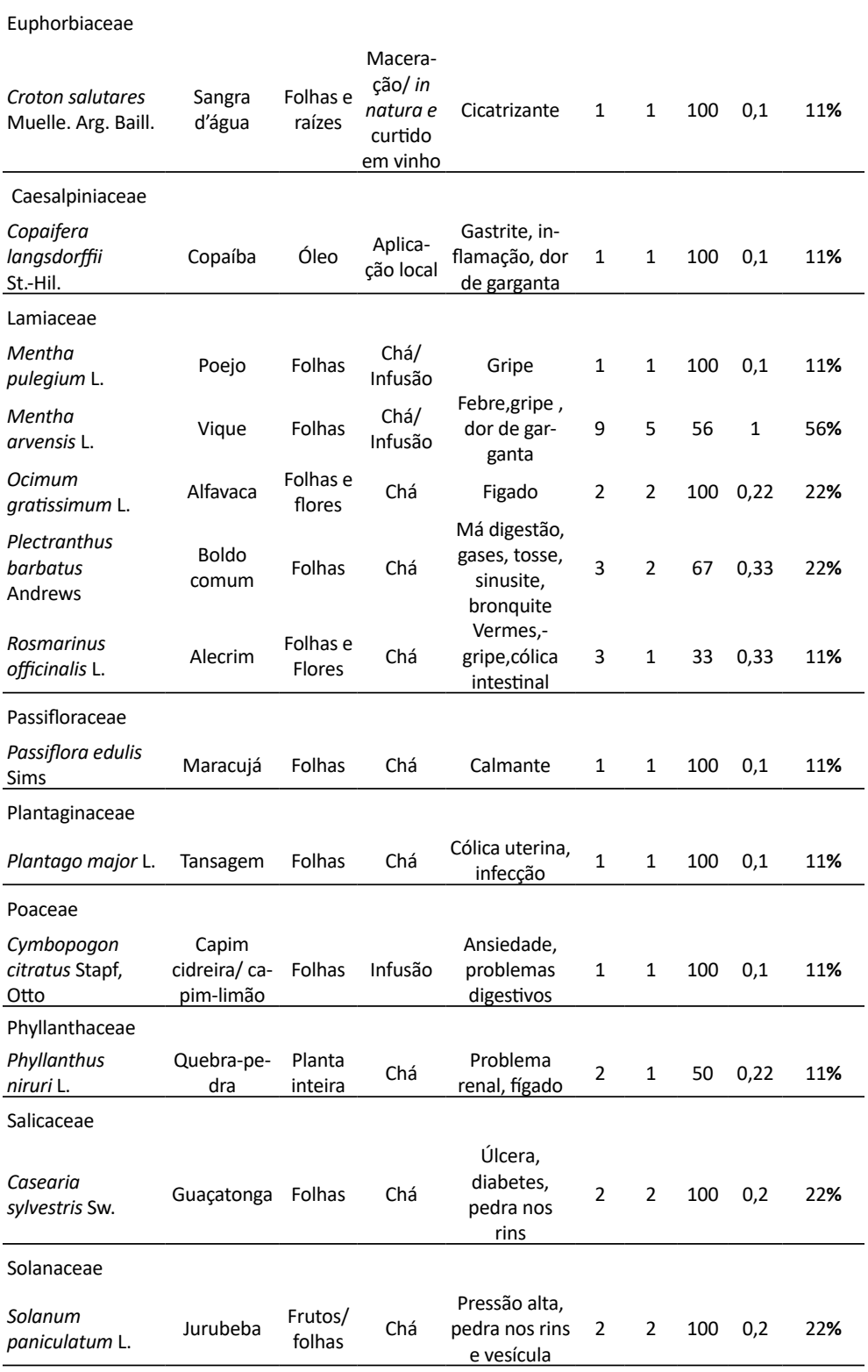




\begin{tabular}{|c|c|c|c|c|c|c|c|c|c|}
\hline \multicolumn{10}{|l|}{ Verbenaceae } \\
\hline $\begin{array}{l}\text { Lippia alba (Mill.) } \\
\text { N.E. Br. ex Britton } \\
\text { \& P. Wilson }\end{array}$ & Erva cidreira & $\begin{array}{l}\text { Folhas/ } \\
\text { semen- } \\
\text { te }\end{array}$ & Infusão & $\begin{array}{c}\text { Dor de } \\
\text { cabeça, } \\
\text { enxaqueca, } \\
\text { ansiedade }\end{array}$ & 3 & 2 & 67 & 0,33 & $22 \%$ \\
\hline \multicolumn{10}{|l|}{ Zingiberaceae } \\
\hline $\begin{array}{l}\text { Alpinia zerumbet } \\
\text { (Pers.) B.L. Burtt } \\
\text { \& R.M. Sm. }\end{array}$ & Colônia & Folhas & Chá & Artrite, asma, & 2 & 1 & 50 & 0,22 & $11 \%$ \\
\hline $\begin{array}{l}\text { Zingiber officinale } \\
\text { Roscoe }\end{array}$ & Gengibre & Raíz & Chá & $\begin{array}{l}\text { Anemia, } \\
\text { tosse, má } \\
\text { digestão }\end{array}$ & 2 & 1 & 50 & 0,22 & $11 \%$ \\
\hline Curcuma longa $\mathrm{L}$. & $\begin{array}{l}\text { Açafrão da } \\
\text { terra }\end{array}$ & $\begin{array}{c}\text { Raíz/ } \\
\text { Rizoma }\end{array}$ & Chá & $\begin{array}{l}\text { Dor de gar- } \\
\text { ganta, tosse }\end{array}$ & 2 & 1 & 50 & 0,22 & $11 \%$ \\
\hline $\begin{array}{l}\text { Curcuma zedoaria } \\
\text { Roscoe, William }\end{array}$ & Zedoária & Rizoma & Chá & $\begin{array}{c}\text { Gastrite, mau } \\
\text { hálito }\end{array}$ & 2 & 1 & 50 & 0,22 & $11 \%$ \\
\hline
\end{tabular}

Tabela 2 - Espécies medicinais usadas pelos entrevistados do Horto Florestal. Cuiabá -MT, 2017.

Abreviações: FSP = Frequência absoluta dos informantes que citaram a espécie; FID = Frequência absoluta dos informantes que citaram os usos principais; NF = Nível de fidelidade; FC = Fator de correção; PCUSP = frequência relativa de concordância quanto aos usos principais

Fonte: Construção dos Autores

A folha (12 citações) é a parte da planta mais utilizada pelos depoentes no preparo dos remédios, seguido da flor, raiz e rizoma (duas citações cada). Resultados semelhantes são encontrados nos trabalhos de Jesus et al (2009); Oliveira (2012), Freitas et al (2012); Mamede e Pasa (20140 e ressaltam que as folhas são as mais usadas devido ao porte herbáceo e por possuírem folhas disponíveis o ano todo (Figura 8).

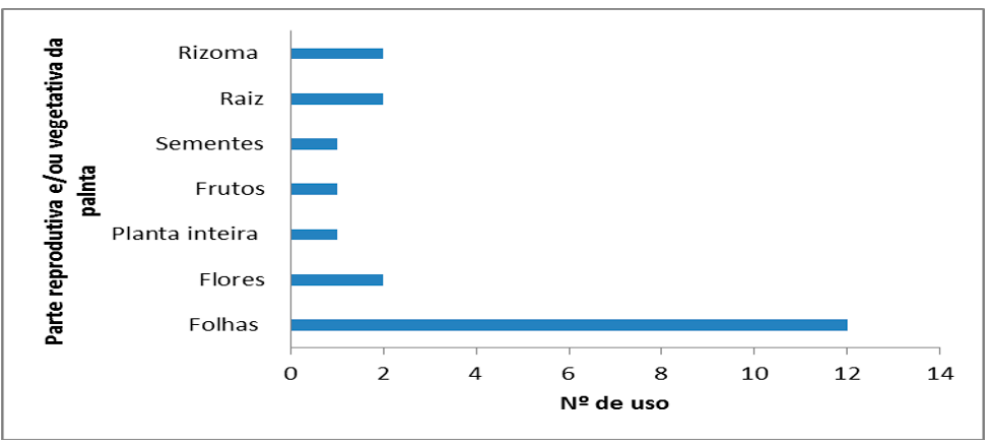

Figura 8. Partes da planta usadas como remédio. Horto Florestal. MT, 2017 Fonte: Construção dos Autores 
Ao referirem sobre as formas de usos, o chá teve maior destaque, com um total de 21 citações, seguido pela infusão (seis citações). Dados semelhantes foram publicados no estudo de Pasa e Mamede (2014), na Comunidade de São Miguel, zona rural de Várzea Grande-MT, conforme Figura 9.

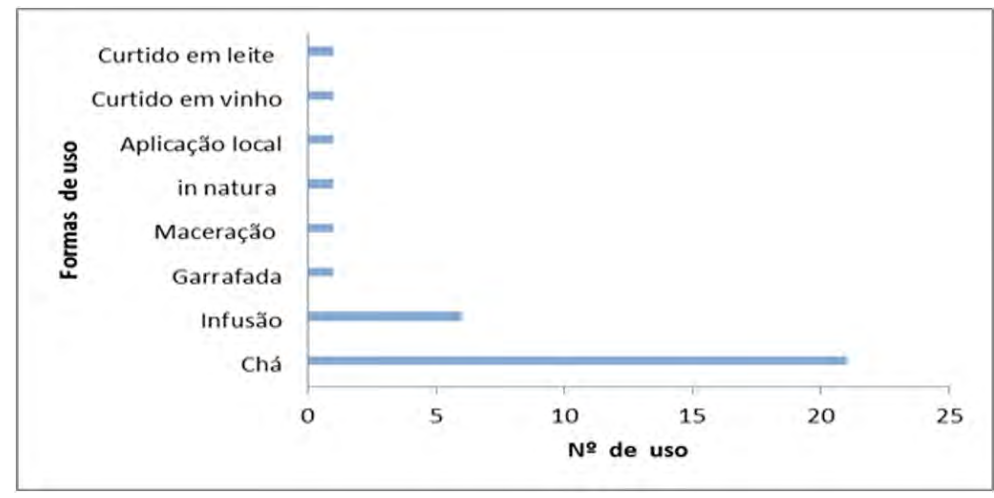

Figura 9. Formas de preparo das espécies medicinais. Horto Florestal. MT, 2017 Fonte: Construção dos Autores.

Pode-se constatar na Tabela 3, que diferentes partes de uma mesma planta foram mencionadas para usos distintos. Podemos citar como exemplo a carqueja; camomila; sangra d'água; alfavaca; alecrim; jurubeba; erva cidreira e açafrão da Terra. Este fato evidencia a importância de levantamentos etnobotânicos onde há o registro da parte da planta utilizada, pois diferentes estruturas, vegetativas e/ou reprodutivas podem potencializar um constituinte químico distinto (SANTOS, 2003). 


\begin{tabular}{lcc}
\hline Espécie & Nome vernacular & Parte utilizada \\
\hline Bacharis trimera & Carqueja & Folhas e raízes \\
Matricaria reticulita & Camomila & Folhas e flores \\
Croton salutares & Sangra d'água & Folhas e raízes \\
Ocimum gratissimum & Alfavaca & Folhas e flores \\
Rosmarinus officinalis & Alecrim & Folhas e flores \\
Solanum paniculatum & Jurubeba & Frutos e folhas \\
Lippia alba & Erva cidreira & Folhas e semente \\
Curcuma longa & Açafrão da terra & Raíz e rizoma \\
\hline
\end{tabular}

Tabela 3. Partes da planta usadas como medicinais. Horto Florestal. Cuiabá-MT, 2017

Fonte: Construção dos Autores

\section{Cultivo e manejo das espécies vegetais}

A horta está instalada em uma área de cultivo com aproximadamente $4.000 \mathrm{~m}^{2}$, circundada por mata ciliar com espécies nativas e exóticas, conforme Figura 10.
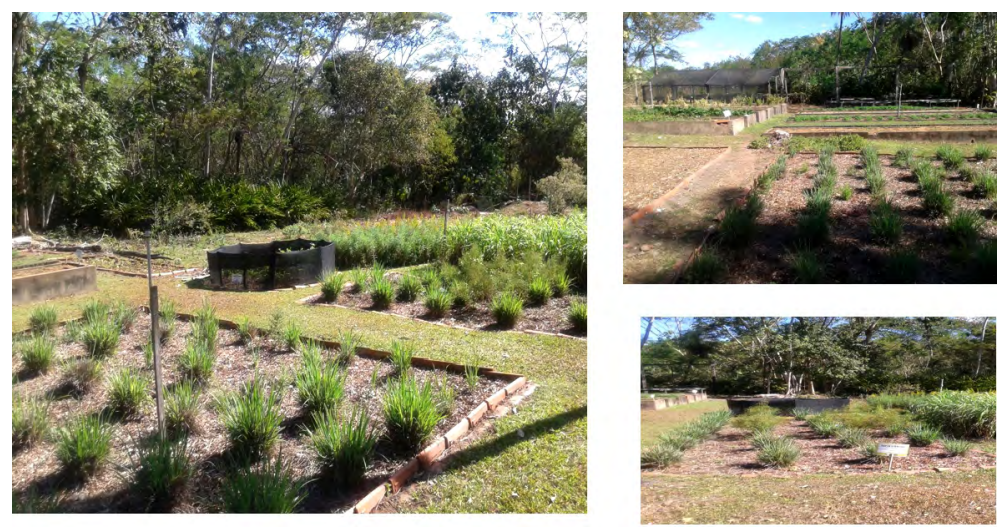

Figura 10. Área do viveiro de plantas Medicinais. Horto Florestal. MT, 2017 Fonte: Acervo dos Autores

Os canteiros que compõe este mosaico são em alvenaria e estão dispostos em formato de mandala (círculo) e de linha reta, em posição Leste-Oeste (Figura 11). 

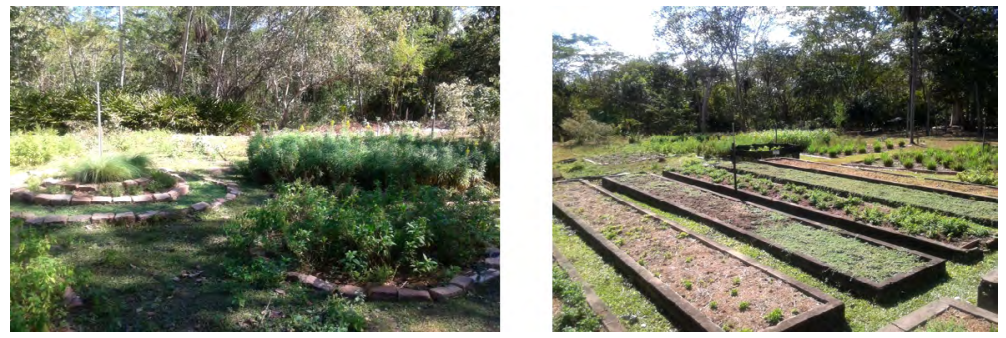

Figura 11.Vista geral do formato dos canteiros do viveiro. Horto Florestal. MT, 2017 Fonte: Acervo dos Autores

De acordo com Azevedo (2010), ao se cultivar plantas de origem medicinal, alguns fatores devem ser observados:

"... o aumento da temperatura faz aumentar também a velocidade de crescimento da planta. Luz - desempenha papel fundamental na vida das plantas, influenciando na fotossíntese, como crescimento, desenvolvimento e forma das plantas. Umidade - A deficiência de água no solo ("stress" hídrico) pode aumentar ou diminuir os princípios ativos de acordo com a cultivar estudada. Altitude - à medida que aumenta a altitude (acima de 100 metros), a temperatura diminui 1 1ㄷ $\mathrm{C}$ e aumenta a insolação, que interfere no desenvolvimento das plantas e na produção de princípios ativos. Plantas produtoras de alcalóides, quando em baixas altitudes, apresentam maior teor de princípios ativos. Latitude - teoricamente, plantas cultivadas em latitudes equivalentes (Norte e Sul) têm o mesmo desenvolvimento, época de floração e teor de princípios ativos (...)

A área do viveiro destinada ao cultivo de plantas medicinais, constitui-se de um solo originalmente arenoso. Para a produção de adubo orgânico, foi separada uma área destinada ao preparo deste, cujo os elementos obtem-se a partir do esterco bovino, palha de arroz e do material vegetal oriundo da própria horta e os insumos resultantes das podas e dos arredores, ocorre também à adubação com torta de mamona e micronutrientes (Figura 12). Sempre é feito o revolvimento e incorporação do material orgânico curtido, visando manter a umidade e absorção de nutrientes ao solo. De acordo com Azevedo (2010) o adubo orgânico é todo produto proveniente da decomposição de resíduos de origem animal e vegetal com elevados teores de componentes orgânicos. 

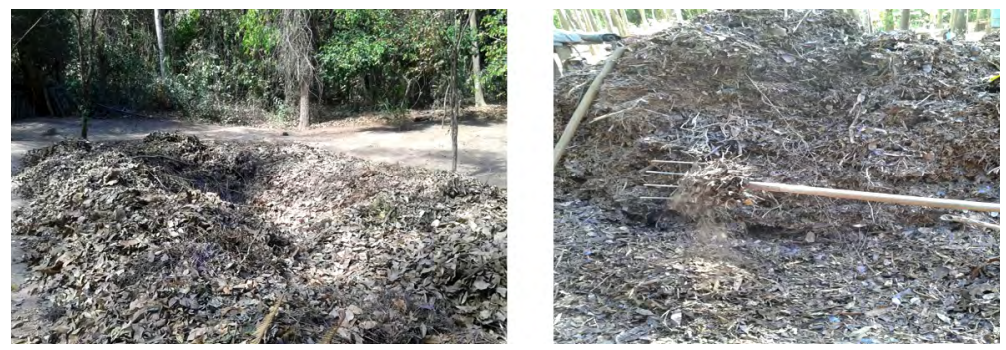

Figura 12 - Área de produção de adubo orgânico. Horto Florestal. MT, 2017 Fonte: Acervo dos Autores

Muitas ervas medicinais que surgiram espontaneamente na horta foram mantidas. Uma área (uma parte dos canteiros) foi destinada à manutenção de plantas matrizes adquiridas na comunidade, sobretudo de quintais de moradores tradicinais como é caso do morador Antônio, que reside em Cuiabá há mais de 50 anos e, que cultiva espécies medicinais em uma área de sua residência. Tal cuidado, visa assegurar um exemplar fidedigno, e livre de agrotóxicos.

As plantas que compõem o viveiro estão postas de forma estratégica, algumas espécies são plantadas próximas e/ou como bordadura, pois exercem função herbicida ou agem como repelente natural, como é caso da hortelã e do poejo. A losna possui um cheiro que repele lepidóptero e protege contra formigas, porém a sua vizinhança não faz bem para nenhuma planta. A alfavaca apresenta cheiro que repele moscas e mosquitos. A arruda funciona como um repelente, porém não deve ser plantada próxima da alfavaca (AZEVEDO, 2010).

\section{CONSIDERAÇÕES FINAIS}

As plantas medicinais fazem parte da cultura popular e o seu uso representa uma fonte de acesso em potencial para as pesquisas de cunho farmacológico, evidenciadas a partir de levantamentos etnobotânicos que permitem a elucidação dos usos para diversos tratamentos. Em sincronia com a etnofarmacologia, que visa à exploração científica do uso tradicional das plantas e como produto o desenvolvimento de novos fármacos.

O manejo e cultivo das plantas do viveiro ocorrem de forma sustentável, sem uso de pesticidas, visando à conservação 
da biodiversidade, e a integridade da flora. Além disso, o Horto é uma Farmácia Viva, que interage com a população, por meio de palestras de educação ambiental, para difundir o conhecimento e alertar acerca dos modos de uso das plantas, tornando-se como referência a comunidade local.

Novos estudos nesta área e que contribuam para o conhecimento dos compostos ativos e possíveis efeitos adversos, a fim de assegurar a confiabilidade no tratamento de doenças em geral, são bem-vindos ao universo das Ciências.

\section{REFERÊNCIAS}

AMOROZO, M. C. M.; GELY, A. Uso de plantas medicinais por caboclos do Baixo Amazonas. Barcarena, PA, Brasil. Boletim Museu Paraense Emílio Goeldi. Sér. Bot. 4, p. 47-131, 1988.

BRASIL. Ministério da Saúde. Gabinete do Ministro. Portaria no 2.488, de 21 de outubro de 2011. Aprova a Política Nacional de Atenção Básica, estabelecendo a revisão de diretrizes e normas para a organização da atenção básica, para a Estratégia Saúde da Família (ESF) e o Programa de Agentes Comunitários de Saúde (PACS). Diário Oficial da União, seção 1, no 204.

CARVALHO, J., C., T. SARTI, S. J.; Fitoterapia e fitoterápicos. In: CARVALHO, J., C., T. Fitoterápicos anti-inflamatórios: aspectos químicos, farmacológicos e aplicações terapêuticas. Ribeirão Preto, SP: Tecmedd, p. 13 - 38, 2004.

CASAGRANDE, Alana. Plantas medicinais e ritualísticas utilizadas pela comunidade do Morro da Cruz. 2009. 90 f. Monografia (Graduação) Universidade Federal do Rio Grande do Sul, Instituto de Biociências. Porto Alegre-RS. 2009.

COSTA, A. F. Farmacognosia: volume II. 6. ed. Lisboa: Fundação Calouste Gulbenkian, ed. 2002. P. 1030.

DE DAVID, M.; PASA, M. C. As plantas medicinais e a etnobotânica em Várzea Grande, MT, Brasil. Interações, v. 16, n. 1, 2015.

DE DAVID, M.; PASA, M. C. Saber popular e as plantas medicinais em Várzea Grande, MT, Brasil. FLOVET, n. 5, 2013.

DOS SANTOS MAMEDE, J. S.; PASA, M. C. Plantas medicinais utilizadas pela comunidade São Miguel, zona rural de Várzea Grande, Mato Grosso, Brasil. FLOVET. 1, n. 6, 2014.

FREITAS, A. V. L. Plantas medicinais: um estudo etnobotânico nos quintais do Sítio Cruz, São Miguel, Rio Grande do Norte, Brasil. Revista Brasileira de Biociências, v. 10, n. 1, p. 48, 2012.

FRIEDMAN, J.; YANIV, Z.; DAFNI, A. \& PALEWITCH, D. A. A preliminary classification or the healing potential of medicinal plants based on a rational analysis of 
an etnopharmacological field survey among Beduins in the Negev desert, Israel. Journal of Ethnopharmacology, 16, 275-287, 1986.

GIRÃO, M.; RODRIGUES, A. G. Diagnóstico situacional de programas estaduais e municipais de fitoterapia, 2005.

GONÇALVES, K. G.; PASA, M. C. A etnobotânica e as plantas medicinais na Comunidade Sucuri, Cuiabá, MT, Brasil. Interações, v. 16, n. 2, p. 245, 2015.

GUARIM NETO, G.; MORAIS, R. G. de. Recursos medicinais de espécies do Cerrado de Mato Grosso: um estudo bibliográfico. Acta Botanica Brasílica, v. 17, n. 4, p. 561-584, dez 2003.

LORENZI, H.; MATOS, F.J.A. Plantas medicinais do Brasil: nativas e exóticas. Nova Odessa, Plantarum. 2002. 544p.

MARIUTTI, L. R.; BRAGAGNOLO, N. B. Revisão: antioxidantes naturais da família Lamiaceae - aplicação em produtos alimentícios. Revista Brasileira de Farmacognosia, 17(2): 96-103. 2007

MARSARO, C. C. S. Perfil sociodemográfico e o impacto ambiental causado pelos visitantes no Horto Florestal em Cuiabá -MT. Trabalho apresentado no IV Congresso Brasileiro de Gestão Ambiental Salvador/BA - 25 a 28/11/2013. Anais... Disponível em http:// http://www.ibeas.org.br/congresso/Trabalhos2013/VI-028. pdf.> Acesso em: 12 Jun. 2017.

MATOS, F. J. A. Farmácias vivas: sistema de utilização de plantas medicinais projetado para pequenas comunidades. 3. Ed. Fortaleza: EUFC, 1998.

MEIHY, J. C. S. B. Manual de História Oral. Ed. Loyola. S. P. 2010.

MINAYO, M. C. de S. (org.). Pesquisa Social: Teoria, Método e Criatividade. Petrópolis: Vozes, 1994.

Ministério da Saúde 2008. Relação Nacional de Plantas Medicinais de interesse para o SUS. Available on http:// portal.saude.gov.br/portal/arquivos/pdf/RENISUS.pdf. Accesso12 Jun. 2017.

OLIVEIRA, A. F. M.; ANDRADE, L. de H. C. Caracterização morfológica de Justicia pectoralis Jacq. e J. gendarussa Burm. F.(Acanthaceae). Acta Amazónica, v. 30, n. 4, p. 569-569, 2000.

OLIVEIRA, C. de. Levantamento etnobotânico na comunidade Bola Verde, Teofilândia, Bahia. 2012. 54 f. Monografia (graduação) - Universidade Federal do Recôncavo da Bahia, Centro de Ciências Agrárias, Ambientais e Biológicas. Cruz das Almas, BA, 2012.

PASA, M. C. Um olhar etnobotânico sobre as comunidades do Bambá, Cuiabá, MT. Ed. Entrelinhas, Cuiabá, MT. 176 p. 2007.

PASA, M. C.; DE DAVID, M.; SÁNCHEZ, D. C. M. Copaifera langsdorffii Desf: Aspectos Ecológicos e Silviculturais na Comunidade Santa Teresa. Cuiabá, MT, Brasil. Biodiversidade, v. 11, n. 1, p. 13-22, 2012. 
PASA, M. C.; GONÇALVES, K. G. Abordagem Etnobotânica de Moringa oleífera Lam.: do cultivo ao uso da espécie em Rondonópolis, Mato Grosso. FLOVET v. 1, n. 1, 2010.

PASA, M. C. Saber local e medicina popular: a etnobotânica em Cuiabá, Mato Grosso, Brasil. Boletim do Museu Paraense Emílio Goeldi. Ciências Humanas, v. 6, n. 1, p. 179-196, jan.-abr. 2011.

SIMÕES, C. M. O.; MENTZ, L. A.; SCHENKEL, E. P.; IRGANG, B. E.; STEHMANN, J. R. Plantas da medicina popular no Rio Grande do Sul. Porto Alegre: UFRG, 173 p., 1988.

SOUZA, S. M. C. Avaliação de óleos essenciais de condimentos sobre o desenvolvimento micelial de fungos associados a produtos de panificação. Ciência e Agrotecnologia, v.28, n.3, p.685-90, 2004. 